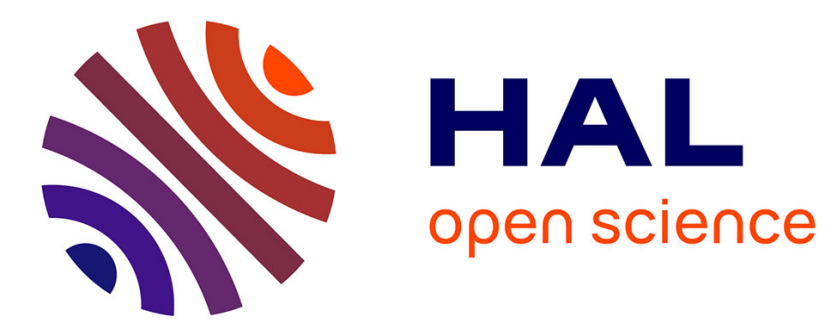

\title{
Quasilinear nature of L mode edge turbulent transport in fluid nonlinear simulations
}

G de Dominici, G. Fuhr, C. Bourdelle, P. Beyer, X. Garbet, Y. Sarazin, G. Falchetto, Contributors Jet

\section{- To cite this version:}

G de Dominici, G. Fuhr, C. Bourdelle, P. Beyer, X. Garbet, et al.. Quasilinear nature of L mode edge turbulent transport in fluid nonlinear simulations. Nuclear Fusion Journal of Plasma Physics and Thermonuclear Fusion, 2019. cea-02375494

\section{HAL Id: cea-02375494 https://hal-cea.archives-ouvertes.fr/cea-02375494}

Submitted on 22 Nov 2019

HAL is a multi-disciplinary open access archive for the deposit and dissemination of scientific research documents, whether they are published or not. The documents may come from teaching and research institutions in France or abroad, or from public or private research centers.
L'archive ouverte pluridisciplinaire HAL, est destinée au dépôt et à la diffusion de documents scientifiques de niveau recherche, publiés ou non, émanant des établissements d'enseignement et de recherche français ou étrangers, des laboratoires publics ou privés. 


\title{
Quasilinear nature of $\mathrm{L}$ mode edge turbulent transport in fluid nonlinear simulations
}

\author{
G. De Dominici ${ }^{1}$, G. Fuhr ${ }^{2}$, C. Bourdelle ${ }^{1}$, P. Beyer ${ }^{2}$, X. \\ Garbet $^{1}$, Y. Sarazin ${ }^{1}$, G. L. Falchetto ${ }^{1}$ and JET Contributors* \\ 1CEA, IRFM, F-13128 St-Paul-Lez-Durance, France \\ ${ }^{2}$ Aix-Marseille Université, CNRS, PIIM UMR 7345, 13397 Marseille Cedex 20, France \\ *See the author list of Overview of the JET preparation for Deuterium-Tritium \\ Operation by E. Joffrin et al. to be published in Nuclear Fusion Special issue: \\ overview and summary reports from the 27th Fusion Energy Conference \\ (Ahmedabad, India, 22-27 October 2018) \\ E-mail: author.one@mail.com
}

\begin{abstract}
.
In tokamak plasma, the edge region is characterized by larger gradients, higher resisitivity and distance to the critical $\beta$ shorter compared to core plasma. By edge, here we mean the region where the pedestal forms when transsiting from $\mathrm{L}$ to $\mathrm{H}$-mode: $r / a=0.85-1$. This paper shows, using a three-dimensional electromagnetic fluid turbulent transport model, that the quasilinear hypothesis is valid in the edge region. The diamagnetic coupling is stabilizing while $\beta$ is destabilizing. The effect of $\beta$ on the characteristic times of turbulence is studied, as its effect on the Reynolds stress and the Maxwell stress in these fluid simulations.
\end{abstract}

PACS numbers: $00.00,20.00,42.12$

Keywords: Article preparation, IOP journals Submitted to: J. Phys. A: Math. Gen.

\section{Introduction}

The high-confinement mode (H-mode) of magnetically confined plasmas has been envisioned as the operating mode for future burning plasmas for its higher global energy confinement and plasma pressure. The transition from low-confinement mode (L-mode) to H-mode occurs at a critical power which depends on various parameters, such as the magnetic field, the density, the plasma surface, the magnetic configuration etc $[1,2]$. Multi-machine databases of the power threshold of the $\mathrm{L}$ to $\mathrm{H}$ transition with respect to engineering parameters have been constructed. This allows estimating the power threshold with scaling laws such as in Martin et al. [3], but there remain high uncertainties in the prediction. Indeed, for a given predicted threshold, the measured 
one can vary by a factor 5 [3]. Moreover, this global approach of the transition is difficult to compare with theoretical work based on local mechanisms.

In order to reduce uncertainties on the threshold power, one needs to understand the drive of turbulence at the L-mode edge prior to the transition in H-mode. As a first step, one can study the dominant(s) instability(-ies) in these regions, characterized by large gradients: $R / L_{p}=50 \rightarrow 120$ (where $R$ is the major radius and $L_{p}$ the pressure gradient length) and high collisionality: $\nu_{*}=0.1 \rightarrow 120$, compared to the core plasma, where $R / L_{p}=5 \rightarrow 15$ and $\nu_{*}=0 \rightarrow 0.1$. Studies with linear fixed gradient gyrokinetic simulations in the edge region $(\rho=0.95-1)[4,5,6]$ show that ballooned resistive modes can be destabilized by the larger edge resistivity compared to the core. These regions also have been studied using fluid models and an electrostatic assumption, using a drift wave only paradigm [7] or an interchange only paradigm [8], or accounting for both couplings with electromagnetic assumption as reported in [9,12,11]. In [11], a competition between the interchange instability and the drift wave instability was mentioned; the latter said to be dominant in nonlinear regime in particular at realistic edge resistivity. Moreover, in these regions, large gradients $R / L_{p}$ leads to low critical $\beta$ above which kinetic ballooning modes are destabilized, known as the ideal MagnetoHydro-Dyname (MHD) boundary [12]. A criteria of this limit is given in [12] in terms of $\nabla \beta$, showing the destabilizing effect of $\beta$. Here some parametric dependencies of the destabilizing effect of $\beta$ are illustrated. Indeed, even if $\beta$ is low in the edge, it is usually not too far from the critical $\beta$, therefore magnetic perturbations play a role in this region. Various more recent fluid models study the MHD equations using more realistic geometry and including the Scrape Off Layer, in 2D with HESEL [12] or in 3D with TOKAM3X [13], but in both cases using an electrostatic assumption.

Here it is proposed to study the plasma edge turbulence using the fluid nonlinear code EMEDGE3D accounting for magnetic fluctuations and both interchange and diamagnetic coupling [14]. EMEDGE3D models the confined plasma, from the LCFS to a few centimeters inside the plasma, with a circular shape. This model has been used in $[15,16]$ but with an electrostatic assumption and neglecting the diamagnetic coupling. In $[15,16]$ it has been shown for the first time that accounting for the neoclassical relaxation of the radial electric field to its equilibrium value, a successful spontaneous edge transport barrier could be generated in 3D flux-driven turbulence simulations. Nonetheless EMEDGE3D, being a fluid code, by definition, over-estimates the saturated state of turbulence compared to gyro-kinetic models. Therefore, in fluid flux driven simulations, the power threshold is under-estimated. However, the goal of such a model is to analyse qualitatively the underlying physics, with parameters as realistic as possible, not to predict the power threshold quantitatively. Following [11], EMEDGE3D has been improved by implementing the electromagnetic effects [14], and more recently the diamagnetic coupling, shown to be essential ingredients in the edge resistive region in previous works $[9,12,11]$. Indeed, at realistic resistivity, $\beta$ is shown to have a destabilizing effect while the diamagnetic coupling has a stabilizing effect. These fluid simulations are performed using a fixed gradient drive, the aim being to be 
as close as possible to edge conditions just prior an H-mode (high collisionality and high pressure gradient) to study the linear and the nonlinear states. These simulations show that the dynamic associated to the nonlinear state stays close to what could happen in a quasilinear simulation.

Hence, the nonlinear simulations presented here show that at the highest resistivity, accounting for diamagnetic coupling or not does not change the nature of the unstable modes and the estimated turbulent flux, whereas at lower, more realistic resistivity, the diamagnetic coupling is stabilizing. The novelty of our work is that a similar behavior with respect to resistivity can be obtained by a quasilinear proxy for the turbulent flux, using linear simulations only. Moreover the nonlinear phase shift between the electrostatic potential and the electron pressure is very close to the linear cross-phase at low wave numbers, similarly to what was found in the core [19]. The quasi-linear nature of turbulent transport, meaning that the fluctuating transported quantities respond linearly to fluctuating electroamgnetic potentials, is further demonstrated by comparing the auto-correlation time (i.e. characterizing the lifetime of a turbulent eddy) to the particle flight time (i.e. the time a particle would spend around a turbulent eddy) $[17,18]$. Both times are computed using EMEDGE3D nonlinear simulations, accounting also for electromagnetic effect at realistic edge $\beta$ values. In all cases, we show that the autocorrelation time is shorter than the particle flight time, and really close to the inverse of the instability growth rate. Therefore the nonlinear coupling between the saturated electromagnetic potential and the transported quantities is weak and can be neglected. Indeed the particles are not trapped indefinitely in eddies and they can diffuse with a random walk motion. This is the first time that the quasilinear nature of L-mode edge turbulent transport is investigated. For core parameters, hence lower normalized gradients and lower resistivity, the quasilinear nature of the turbulent transport has been more extensively studied using nonlinear gyrokinetic simulations $[17,19,20]$. Moreover, ongoing work using the gyro-kinetic model of GENE in non-linear cases, using L-mode edge parameters is currently on-going, [Effect of the [...] in ASDEX Upgrade and JETILW] N. Bonanomi to be submitted.

In section 2 we focus on the diamagnetic coupling impact and edge turbulence, EMEDGE3D equations are presented; then typical L-mode edge parameters are used to study the impact of the diamagnetic coupling at various values of resistivity; we also show that the nonlinear turbulent flux can be estimated by a quasilinear approximation. In section 3 we focus on the impact of $\beta$, its value being increased to realistic values and its impact is studied in EMEDGE3D and GENE linearly. The same study is done nonlinearly with fixed gradient EMEDGE3D simulations. We show that a quasilinear approximation of the nonlinear transport stands at low $\beta$, but is less valid for high $\beta$. Finally, the conclusions are drawn in section 4. 


\section{Quasilinear aspect of the turbulent transport driven by the competition between RBM and Drift Waves}

The competition between diamagnetic and interchange coupling has been studied using a non-linear gyro-fluid model [11], showing that the main instability changes depending on the resistivity, going from drift wave dominated (diamagnetic coupling dominates) at low but realistic resistivity, to ballooning dominated at higher resistivity (interchange coupling dominates). This change of nature of the dominant mode with the resistivity has also been illustrated by an analytical model [21], showing that:

- When accounting for diamagnetic coupling, the growth rate increases compared to the interchange only case,

- But the diamagnetic coupling reduces the phase shift from $-\pi / 2$ in the interchange only case towards $-\pi / 4$ with the diamagnetic coupling

- The impact of the diamagnetic coupling is larger at lower and more realistic edge resistivity.

The trends observed in the linear analytical model are recovered also in the EMEDGE3D nonlinear simulations. Here the fluid model implemented in EMEDGE3D is first presented in its electromagnetic and diamagnetic version, then the linear trends are studied numerically using both EMEDGE3D [14] and the gyrokinetic model of GENE code [22,23] and finally, the non-linear impact of the diamagnetic coupling on fluid simulations using EMEDGE3D are studied. The quasilinear approximation is shown to be valid for L-mode edge parameters in our fluid nonlinear simulations.

\subsection{The model of EMEDGE3D}

In the present work, flux-driven electromagnetic edge turbulence is simulated in a 3 dimensional toroidal geometry, using the EMEDGE3D code [14]. The system is governed by the following set of fluid equations:

$$
\begin{aligned}
\partial_{t} \nabla_{\perp}^{2} \phi+\left\{\phi ; \nabla_{\perp}^{2} \phi\right\} & =-C_{1} \nabla_{\|} J-\omega_{D}\left(1+\epsilon_{T}\right) G p+\mu_{\perp} \nabla_{\perp}^{4} \phi \\
\partial_{t} p_{e}+\left\{\phi ; p_{e}\right\} & =-C_{2} \nabla_{\|} J+\delta_{c} G\left(\phi-C_{\text {dia }} p_{e}\right)+\chi_{\perp} \nabla_{\perp}^{2} p_{e} \\
\partial_{t} \psi & =-C_{3} \nabla_{\|}\left(\phi-C_{\text {dia }} p_{e}\right)+\eta_{\text {norm }} J
\end{aligned}
$$

Equation (1) is the equation for the vorticity $\nabla_{\perp}^{2} \phi, \phi$ being the electric potential; equation (2) is the energy balance, $p_{e}$ the electron pressure (and $p=p_{e}+p_{i}$ being the total pressure). The density is constant and uniform: $p_{e 0}=n_{0} T_{e 0}$ with $p_{e 0}$ and $T_{e 0}$ reference values for the electron pressure and temperature. Furthermore, the ratio between ion and electron temperatures is kept constant: $T_{i 0}=\epsilon_{T} T_{e 0}$. Equation (3) 
is Ohms law equation, $\psi$ being the magnetic vector potential. The dimensionless coordinates $(x, y, z)$ of the 3D toroidal geometry correspond respectively to the minor radius, and the poloidal and toroidal angles. The EMEDGE3D code uses a semi-spectral scheme, with the poloidal and toroidal directions being decomposed in Fourier modes, respectively characterized by a poloidal number $m$, and un toroidal number $n$, while the radial direction is treated with finite-differences. Time is normalized to the drift wave time, $\tau_{D W}=L_{p} / c_{s}$, with $c_{s}=\sqrt{p_{e 0} / \rho}$ the isothermal sound speed, $L_{p}$ the pressure gradient length and $\rho$ the mass density. The field $\phi$ is normalized to $C_{\phi}=\frac{B_{0} \xi_{D W}^{2}}{\tau_{D W}}$, the field $p_{e}$ to $C_{p}=\frac{\xi_{D W} p_{e 0}}{L_{p}}$ and the field $\psi$ to $C_{\psi}=\frac{\beta B_{0} \xi_{D W}^{2}}{L_{\|}}$with $L_{\|}=R_{0}$ the parallel scale length. The perpendicular scale length is the ion Larmor radius, $\xi_{D W}=\rho_{i} . J$ is the parallel current defined as $J=\nabla_{\perp}^{2} \psi$. At the boundaries, Dirichlet conditions such that $p, \phi, \psi=0$ are applied to both sides.

$\nabla_{\perp}$ and $\nabla_{\|}$are the perpendicular and parallel gradients, and $\nabla_{\perp}^{2} \phi$ is the vorticity of the $\vec{E} \times \vec{B}$ flow. $G \cdot=\sin \left(\kappa_{y} y\right) \partial_{x} \cdot+\cos \left(\kappa_{y} y\right) \partial_{y}$. is the toroidal curvature operator, and $\{\phi, \cdot\}$ is the usual Poisson bracket: $\{f, g\}=\partial_{x} f \partial_{y} g-\partial_{y} f \partial_{x} g$ which accounts for advection by the $\vec{E} \times \vec{B}$ velocity.

$\nu_{\perp}$ and $\chi_{\perp}$ are the normalized classical viscosity and perpendicular thermal diffusivity. $\quad \eta_{\text {norm }}$ is the normalized resistivity, $\eta_{\text {norm }}=\frac{\eta_{0} \tau_{\tau_{W}}}{\mu_{0} \xi_{D W}^{2}}$ with $\eta_{0}$ the Spitzer resistivity. $\delta_{c}=2 \frac{5}{3} \frac{L_{p}}{R_{0}}=\frac{5}{3} \omega_{D}$ is a geometrical parameter characterizing the curvature coupling in the pressure equation. $C_{1}=\frac{v_{a}^{2} \tau_{D W}^{2}}{L_{\|}^{2}} \beta_{X}, C_{2}=\frac{5}{3} \frac{\tau_{D W} v_{a}^{2} L_{p}}{L_{\|}^{2} \omega_{c i} \xi_{D W}} \beta_{X}$ and $C_{3}=\frac{1}{\text { betaX }}$ are normalized parameters where $v_{a}$ is the Alfvens speed, $\beta_{X}=\frac{\beta_{e} L_{p}^{2}}{L_{\|}^{2}}$ the normalized beta and $\omega_{c i}$ the ion cyclotron frequency.

In the simulations here, the safety factor $q$ varies between 2.5 and 3.5 in the main simulation domain, and has a hyperbolic profile along the radial direction $\frac{1}{q(x)}=\frac{1}{q_{0}}-\frac{\kappa_{y}}{\kappa_{z}} x$ with $q(0)=q_{0}=3, \kappa_{y}=\frac{\xi_{D W}}{r_{0}}$, and $\kappa_{z}=\frac{L_{\|}}{R_{0}}=1 . r_{0}$ is the minor radius at $q=q_{0}$ and $x=\frac{r-r_{0}}{\xi_{D W}}$. The main simulation domain covers a radial extension between $0.86<r / a<1$ with $a$ the plasma minor radius. It is bounded on both sides by buffer zones. $\chi_{\perp}$ is artificially increased in the buffer zones in order to prevent turbulence growth near the extremal points of the radial domain only in nonlinear simulations.

In the following subsections, two configurations are distinguished: the interchange only configuration, for which $C_{d i a}=0$ and the interchange + diamagnetic configuration, for which $C_{d i a}=1$, hence when both diamagnetic and interchange couplings are accounted for $C_{d i a}=\frac{\tau c_{s}^{2}}{L_{p} \xi \omega_{c i}}=1$ using drift wave normalization). In any case, the initial state is a random phase distribution for the fluctuating fields. A background gradient described in the next subsection is used. Then the system evolves first through a linear phase, and then saturates, as represented on figure 1.

\subsection{Drive of instabilities in EMEDGE3D/GENE}

The impact of the diamagnetic coupling on fluid simulations is studied using EMEDGE3D as detailed in section 2.1 in its linear version. This means that $f, g=$ 


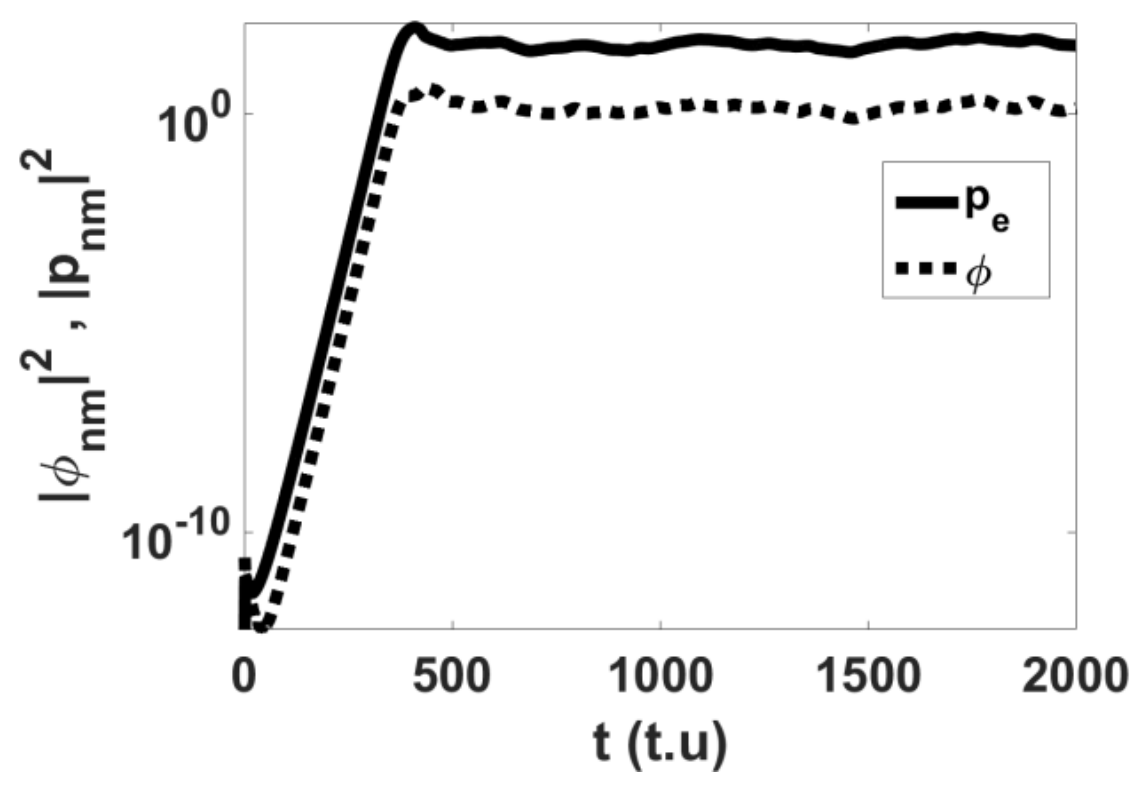

Figure 1. Time traces of the half squared amplitudes of $\tilde{\phi}$ (dotted) and $\tilde{p}_{e}$ (full line) for a typical run of EMEDGE3D, showing first the linear state, and saturation starting from $t=500$.

Table 1. L-mode edge like parameter set used with EMEDGE3D.

\begin{tabular}{llllllll}
\hline$T_{i 0}(\mathrm{eV})$ & $T_{e 0}(\mathrm{eV})$ & $n_{0}\left(\mathrm{~m}^{3}\right)$ & $B_{0}(T)$ & $\eta_{0}(\Omega m)$ & $\beta$ & $\frac{R}{L_{p}}$ & $q$ \\
\hline 40 & 40 & $5 \cdot 12^{19}$ & 1 & $2.6 \cdot 12^{-6}$ & $0.32 \%$ & 58 & $2.5 \rightarrow 3.5$ \\
\hline
\end{tabular}

Table 2. L-mode edge like parameter set used with GENE.

\begin{tabular}{lllll}
\hline$T_{e 0, G}(e V)$ & $n_{0, G}\left(m^{3}\right)$ & $B_{0}(T)$ & $\eta_{0, G}(\Omega m)$ & $\beta_{G}$ \\
\hline 150 & $4 \cdot 12^{19}$ & 3 & $4.2 \cdot 12^{-7}$ & $0.16 \%$ \\
\hline$\frac{R}{L_{n}}$ & $\frac{R}{L_{T}}$ & $k_{\theta} \rho$ & $q$ & $\rho$ \\
\hline 20 & 70 & 0.1 & 3.5 & 0.97 \\
\hline
\end{tabular}

$\partial_{x} f \partial_{y} g-\partial_{y} f \partial_{x} g={ }_{l i n} \partial_{x} f_{00} \partial_{y} g-\partial_{y} f \partial_{x} g_{00}$ with $f, g$ 3D fields. Simulations are performed using a fixed gradient drive $\left\{\phi, p_{e}\right\}=-g_{p} \partial_{y} \phi$ with $g_{p}$ the magnitude of the gradient, here $g_{p}=1$. The other parameters can be computed using table 1 , characteristic of L-mode edge parameters: large pressure gradients length $R / L_{p}$, large resistivity due to low temperatures in these regions and large $q$ values, see for example [5] for edge parameters based on DIII-D and Tore Supra, and [4] for JET like parameters.

Parameters used with EMEDGE3D can be found in table 1, while JET-like parameters used in GENE are given in table 2.

For all simulations presented in section 2 and 3, we use 192 points in the radial domain, with $d x=0.58$. The spectral domain is defined by $\left(m_{\max } ; n_{\max }\right)=(132 ; 32)$ 
with $d n=4$. Here, $k_{\theta} \rho=m \kappa_{y}$ with $\kappa_{y}=\xi_{D W} / r_{0}=0.002$ using parameters of table 1 meaning that $k_{\theta} \rho \in[0 ; 0.264]$.

All parameters are close, especially the gradients lengths, except the resistivity which is lower in the JET-like case. But a scan of 4 resistivity values around the nominal value given in table 1 is performed with EMEDGE3D: $\eta_{0} / 12, \eta_{0}, 12 \eta_{0}, 20 \eta_{0}$. Then the JET like resistivity is recovered near $\eta_{0} / 12$. Figure 2 represents an estimation of a quasilinear flux for the 4 resistivity values, for the interchange only configuration in blue, and the case interchange + diamagnetic configuration in dashed yellow.

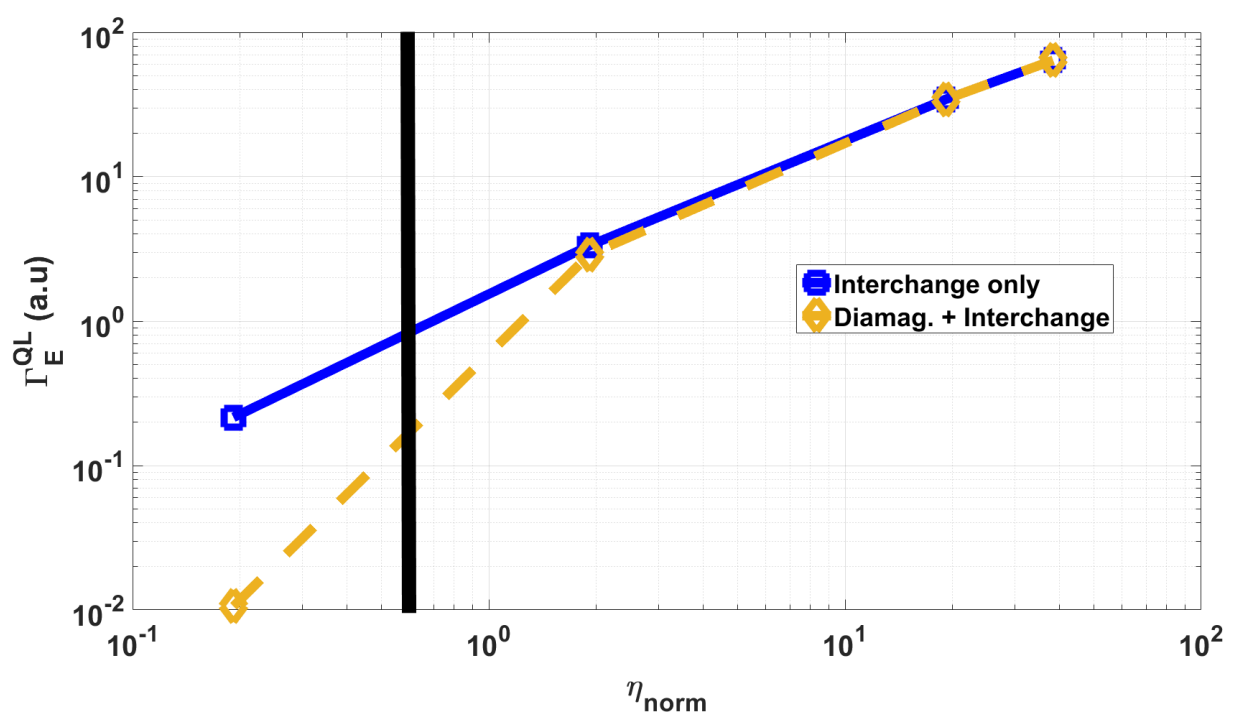

Figure 2. Quasilinear approximation of the turbulent flux $\Gamma_{E}^{Q L}$ (a.u) depending on the resistivity. The yellow dashed line represents the interchange + diamagnetic configuration $\left(C_{d i a} \neq 0\right)$. The blue line represents the interchange only configuration $\left(C_{d i a}=0\right)$. The vertical black line represents the resistivity for the JET like case computed with table 2 .

To compute this quasilinear proxy for the turbulent flux, at each value of the normalized resistivity, the maximum growth rate over $k_{y}=k_{\theta} \rho_{i} \in[0 ; 0.26]$ is taken, as well as the phase shift at the corresponding $k_{\perp}$ to estimate a quasilinear turbulent flux, $\Gamma_{E}^{Q L} \propto \frac{\gamma_{\max }}{k_{\perp}^{2}} \sin |\Delta \Phi|[19,36]$. Then $\gamma_{\max }$ is the maximum growth rate at corresponding $k_{\perp}$ and $\Delta \Phi$ the phase shift between $\tilde{p}_{e}$ and $\tilde{\phi}$ for this growth rate. This definition is a 0-order approximation of what could be a quasilinear estimation of the turbulent flux computed with a quasilinear model, inspired from previous work in the core plasma $[19,36]$. It is based on the heuristic mixing length estimate: $\chi_{\perp} \sim D \sim \gamma / k_{\perp}$ with $\chi_{\perp}$ the perpendicular diffusion coefficient, and supposing that the maximum transport is reached at the maximum growth rate. Knowing that if the potential and the quantity transported are in phase, no transport is awaited, we find $\Gamma_{E}^{Q L} \propto \frac{\gamma_{\max }}{k_{\perp}^{2}} \sin |\Delta \Phi|$. It is not computed during the simulations, as only linear simulations are performed here, but it is an a posteriori estimation. We could have used a transport estimations coming from the Ficks law, but it does not take into account the phase shift, which plays an essential 
role here.

For the highest resistivity, accounting for the diamagnetic coupling does not modify the computed quasilinear proxy of the turbulent flux as illustrated on figure 2, whereas at lower and more realistic resistivity, the diamagnetic coupling is stabilizing in our simulations. The JET-like resistivity is represented by the vertical black line.

In EMEDGE3D, the diamagnetic coupling also has an effect on the mode structure, as illustrated on figure 3. This figure represents the radial extension of each poloidal Fourier m-mode for the toroidal mode $n=8$, as an example (this gives $k_{y}=k_{\theta} \rho_{i} \in$ $[0.038 ; 0.064])$.
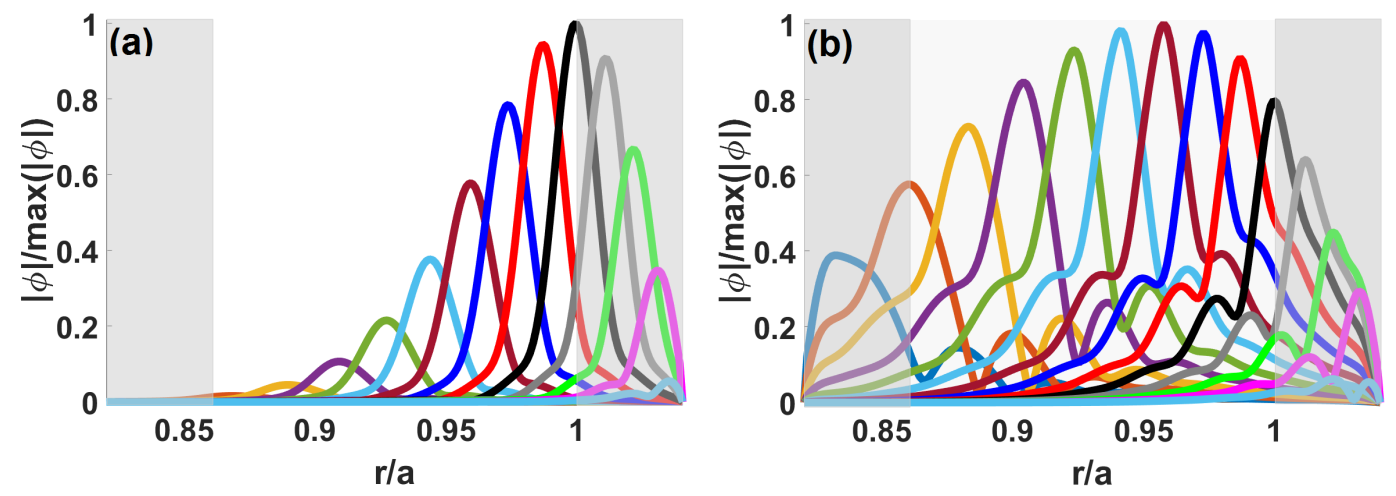

Figure 3. Profile of Fourier components $(m, n)$ of a global mode for $n=8$, at $\eta_{0} / 12$. (a) represents the profile for the interchange only configuration. (b) represents the profile for interchange + diamagnetic configuration. There is no artificial increase of $\chi_{\perp}$ in the buffer zones here.

Modes are ballooned in both configurations. But in the interchange + diamagnetic configuration, the basis of the mode structure is wider due to the diamagnetic coupling which is less localized on the low field side than the interchange control case. The diamagnetic coupling tends to warp the ballooning mode structure.

A similar trend is observed with the gyrokinetic code GENE $[22,40]$ simulations used with JET-like parameters given in table 2. Figure 4 shows the linear growth rate as a function of resistivity. Similarly to the results of EMEDGE3D, the eigenmodes plotted along the poloidal coordinate at moderate resistivity exhibit some shoulders due to the diamagnetic contribution to the resistive modes, figure 4 (c); therefore the unstable modes at realistic resistivity are dominated by resistive drift waves and not resistive ballooning modes $(\mathrm{RBM})$ in these kinds of simulations. Ultimately at low resistivity, the ITG branch is dominated with ballooned eigenvalues as represented in figure 4 (b), which is not reached using EMEDGE3D.

Therefore, in past linear GENE stability analysis carried out in L-mode edge $[5,6]$, the modes with a ballooned structure and shoulder and destabilized by higher resistivity were drift waves modes rather than RBM as initially thought. RBM appear at even larger resistivity than drift waves 4 (d). A quantitative comparison between EMEDGE3D and GENE is not proposed here. Indeed, there are numerous differences 
between the two codes. For example in EMEDGE3D, only the pressure equation is accounted for, then $L_{p}$ is the only input for the gradient length. On the other hand, GENE is a more complete model, where $L_{T}$ (the temperature gradient length) and $L_{n}$ (the density gradient length) are taken into account. Different $\left(L_{n}, L_{T}\right)$ can be used to compute the same $L_{p}$. Nonetheless, in future works, it would be worth studying further the resistivity "threshold" (which is different here between EMEDGE3D and GENE) of the transition "ballooning - drift waves" an its multiple dependencies.
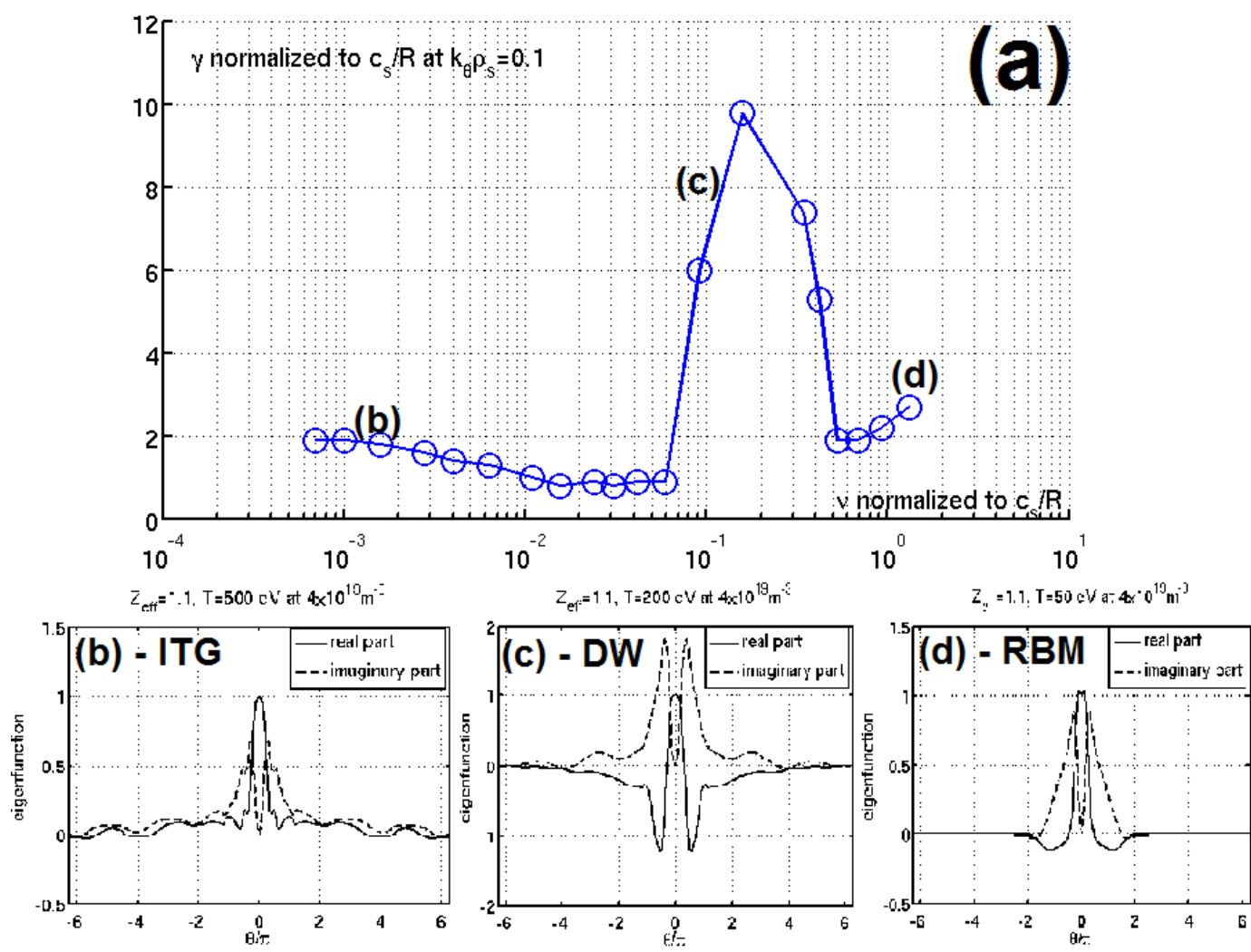

Figure 4. (a) GENE normalized linear growth rate vs normalized resistivity (b) Eigenmode for a resistivity corresponding to $T=500 \mathrm{eV}$ showing a ITG-TEM like mode structure. (c) Eigenmode for a resistivity corresponding to $T=200 \mathrm{eV}$ showing a drift wave like mode structure. (d) Eigenmode for a resistivity corresponding to $T=50$ $\mathrm{eV}$ showing a resistive ballooning mode (RBM) structure. Parameters are JET-like from table 2. The resistivity is scanned around a realistic value corresponding to $T=150 \mathrm{eV}$.

\subsection{Turbulence simulations with diamagnetic effects}

As above, EMEDGE3D is used with the parameters of table 1, using the fully nonlinear Poissons bracket. The resistivity scan is done with the same values of resistivity than linear simulations. Time evolution of the half squared amplitudes of $\tilde{\phi}$ and $\tilde{p}_{e}$ are shown in figure 5 for the interchange + diamagnetic configuration. Figure 5 (a) shows the 
saturated state, and figure 5 (b) shows the pressure fluctuation level in the simulation region as a function of time during this state.


Figure 5. (a)Time evolution of the half squared amplitudes of $\tilde{\phi}$ (dotted) and $\tilde{p}_{e}$ (full line) for the Interchange + Diamagnetic configuration in the saturated state. (b) Pressure fluctuation level in the simulation region as a function of time, on the x-axi, and radius, y-axis.

Here, the electron pressure profile $\bar{p}_{e}(x, t)=\left\langle p_{e}\right\rangle_{y, z}$ evolves self-consistently according to the energy transport equation (the average on a flux surface of equation 2),

$$
\partial_{t} \bar{p}_{e}=-\partial_{x}\left(Q_{\text {coll }}+Q_{\text {conv }}+Q_{\text {mag }}\right)
$$

With $Q_{\text {coll }}=-\chi_{\perp} \partial_{x} \bar{p}_{e}, Q_{\text {conv }}=\left\langle p_{e} \partial_{y} \phi\right\rangle_{y, z}=Q_{E}$ and $Q_{\text {magn }}=-C_{2}\left\langle p_{e} \partial_{y} \psi\right\rangle_{y, z}$.

In figure 6 (a), the nonlinear turbulent flux $Q_{E}$ is plotted versus the resistivity, for the interchange only configuration (full blue line), and the model accounting for both couplings (yellow dashed line).

As obtained in the linear study above, at high resistivity, both configurations give the same nonlinear turbulent flux, but at low resistivity the nonlinear turbulent flux for the interchange only configuration is higher (by an order of magnitude) than the one for the interchange + diamagnetic configuration. This is due to the fact that phase shifts are different: as plot in figure 6 (b), the phase shift $\Delta \Phi$ (divided by $\pi$ ) of the diamagnetic + interchange configuration is always below $-\pi / 2$, characteristic of the drift wave, whereas for the interchange only configuration, it is always at $-\pi / 2$, characteristic of the RBMs, leading to a maximum transport. The nonlinear turbulent flux is normalized to $\frac{C_{p} \xi_{D W}}{\tau_{D W}}=13 \mathrm{kWm} \mathrm{m}^{-2}$. The nonlinear turbulent flux for the JET-like resistivity is $Q_{E, J E T-l i k e} \approx 0.2$ in normalized units, therefore $Q_{E, J E T-l i k e} \approx 2.6 \mathrm{kWm} \mathrm{W}^{-2}$ which is in the right order of magnitude if we compare to experiments.

In [12], a criterion for the transition from drift wave mode to resistive ballooning modes is defined. It is such that if $\nu_{B}=\frac{\nu_{e} L_{\perp}}{c_{s}}>10$ the RBM dominates, $\nu_{e}$ being the electron collision frequency, $c_{s}$ the sound speed and $L_{\perp}$ the perpendicular characteristic length. The values of $\nu_{B}$ for all the resistivity studied here are reported in table 3 . The two cases where $\nu_{B}<10$ correspond to cases where turbulence is drift wave dominated. 

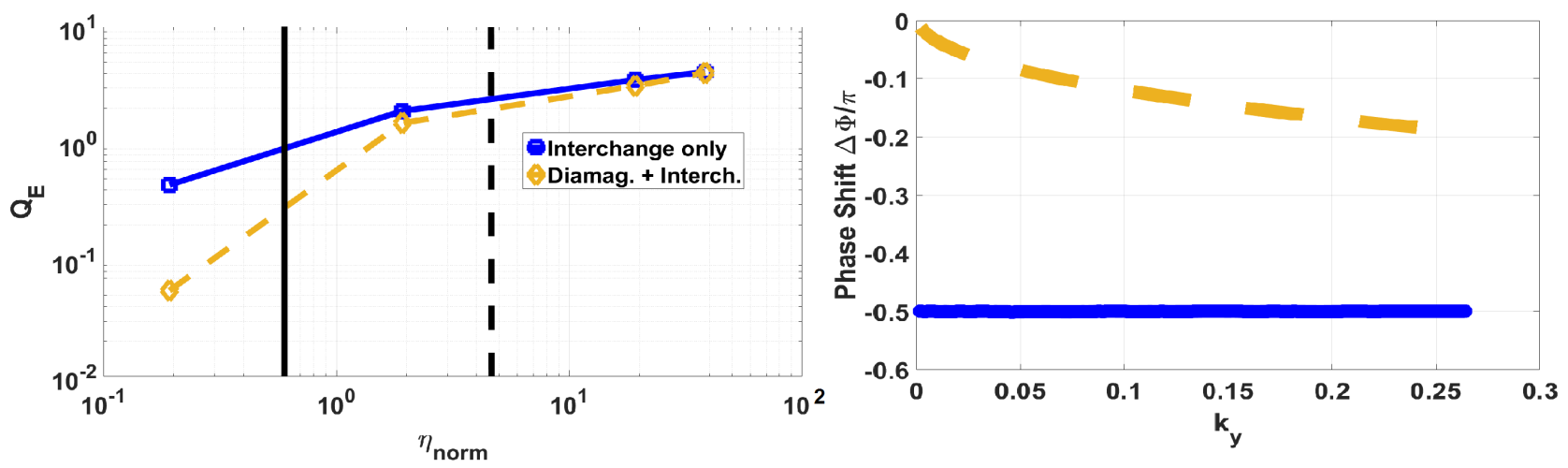

Figure 6. (a) Nonlinear turbulent flux $Q_{E}=\left\langle p_{e} \partial_{y} \phi\right\rangle_{y, z}$ computed for 4 values of resistivity $\left(\eta_{0} / 12, \eta_{0}, 12 \eta_{0}, 20 \eta_{0}\right)$ and the two configurations: the yellow dashed line represents the interchange + diamagnetic configuration and the blue line represents the interchange only configuration. The vertical black line represents an L-mode edge JETlike resistivity, and the vertical dashed black line the critical value given in [12], below which diamagnetic effects have an impact. (b) Spectrum of the phase shift $\Delta \Phi / p i$ for the cases at $\eta_{0} / 10$. In blue, the phase shift for the interchange only configuration is always $-1 / 2$ while for the diamagnetic + interchange configuration is always below.

Table 3. values of $\nu_{B}$ as defined in [12].

\begin{tabular}{lllll}
\hline case & $\eta_{0} / 12$ & $\eta_{0}$ & $12 \eta_{0}$ & $20 \eta_{0}$ \\
\hline$\nu_{B}$ & 1.21 & 5.19 & 24.07 & 38.98 \\
\hline
\end{tabular}

For the two cases where $\nu_{B}>10$, turbulence is RBM dominated. Therefore, the criterion proposed in [12] seems to be roughly valid within EMEDGE3D.
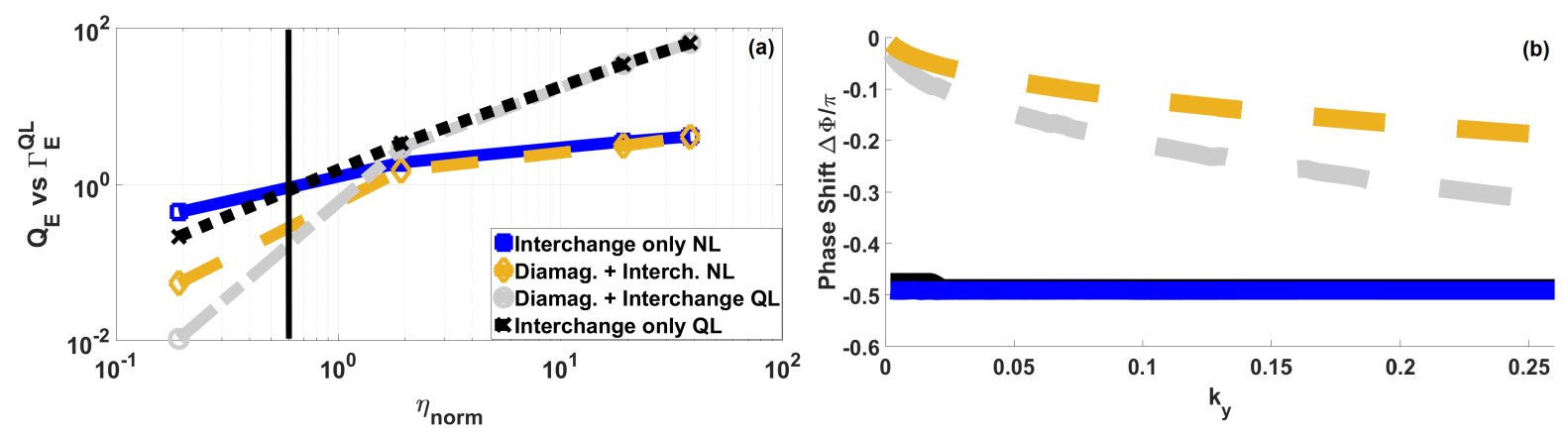

Figure 7. (a) Comparison of the nonlinear turbulent flux $Q_{E}$ (as on figure 6 (a)) and the quasilinear approximation of the turbulent flux $\Gamma_{E}^{Q L}$ (as on figure 2 (a)), plotted with respect to resistivity, for both configurations. (b) Comparison of the phase shift between linear and nonlinear simulations.

The similitudes between the proxy of the quasilinear fluxes $\Gamma_{E}^{Q L}$ presented in figure 2 (a) and the nonlinear fluxes $Q_{E}$ presented in figure 6 (a) are striking, and are overlapped in figure 7 (a). If their amplitudes are not comparable in some cases (for very low and very high resistivity), they behave qualitatively in the same way. Therefore a 
quasilinear proxy for the fluxes is able to correctly grasp the competition between the interchange drive and the diamagnetic coupling as the resistivity varies. Moreover, the linear phase shifts are close between the linear and nonlinear cases as seen on figure 7 (a), in particular for the interchange only configuration. This was not anticipated. Indeed, when approaching the LCFS (here where $\rho \in[0.86 ; 1]$ ) the level of turbulent fluctuations increases, see for example density fluctuation level measured by reflectometer ramping up from $1 \%$ to $10 \%$ in this edge region [29]. It was then thought that the quasi-linear theory would not be respected in this region.

In order to test in more depth the validity of the quasi-linear regime within EMEDGE3D nonlinear simulations, the ratio between the auto-correlation time $\tau_{a c}$ (which is the lifetime of the pattern the particle sense, here turbulent eddies) and a flight time $\tau_{f}$ (which is the eddy turnover time, i.e the time that a particle spend in a turbulent eddy) is estimated. This ratio is referred as the Kubo number. Since the shortest time is the one driving the dynamics, $\mathcal{K} u=\frac{\tau_{a c}}{\tau_{f}}<1$ implies that the system is in a hydro quasi-linear regime. A contratio, $\mathcal{K} u=\frac{\tau_{a c}}{\tau_{f}}>1$ that the system is in a strong non-linear turbulent regime $[18,24]$. Indeed, the stochastic nature of a quasilinear theory can be regarded in terms of random walk phenomenology. In this framework, the latter criterion reflects the need of avoiding that particles undergo to a trapping condition in the resulting field pattern, meaning that a quasilinear theory applies when the particle motion perturbation remains small during $\tau_{a c}$. In this case, corresponding to $\mathcal{K} u<1$, a test particle will follow a turbulent eddy over a distance $\left\langle v_{x}\right\rangle \tau_{a c}$, where $\left\langle v_{x}\right\rangle$ the velocity of fluctuation in the perpendicular direction. After $\tau_{a c}$, the eddy will disappear by definition of $\tau_{a c}$, and the test particle will follow another turbulent eddy over a distance $\left\langle v_{x}\right\rangle \tau_{a c}$, but not necessarily in the same direction. There is then a random walk phenomenology with a diffusion coefficient of $D=\frac{\tau_{a c}<v_{x}>^{2}}{2}$. On the other hand, if $\tau_{a c}$ is very long or infinite, the turbulence is frozen. Then a test particle trapped in an eddy will stay trapped for ever, and then there is no diffusion. In this case, if a particle is not trapped in an eddy, then moving between eddies, it will have a ballistic drift, still not diffusive. This corresponds to the $\mathcal{K} u>1$ case.

The flight time $\tau_{f}$ is defined as $\frac{l_{c}}{\left\langle v_{x}\right\rangle}$ where $l_{c}$ is the radial correlation length and $\left\langle v_{x}\right\rangle$ is the root mean square radial velocity of a test particle in the turbulent field $[17,25]$. The radial correlation length for electrostatic perturbations $l_{c, E S}$ is calculated as the $1 / e$ length of the function $C\left(d_{x}\right)=\frac{\left\langle\phi(x, y, z, t) \phi\left(x+d_{x}, y, z, t\right)\right\rangle}{\left\langle|\phi(x, y, z, t)|^{2}\right\rangle}$ with $\langle\cdot\rangle$ representing the flux surface average. All $n=0$ modes are not included in the calculation, which thus considers only the flux inducing $n>0$ background turbulence. For electrostatic perturbations, $\left\langle v_{x}\right\rangle=\sqrt{\left\langle E_{y}^{2}\right\rangle} / B_{0}$, with the same averaging procedure as $l_{c}$ for consistency, with $B_{0}$ the reference magnetic field that can be found in table 1 . The auto-correlation time for electrostatic perturbations $\tau_{c, E S}$ is taken as the $1 / e$ time of the correlation function $C\left(d_{\tau}\right)=\frac{\left\langle\phi(x, y, z, t) \phi\left(x, y, z, t+d_{\tau}\right)\right\rangle}{\left\langle|\phi(x, y, z, t)|^{2}\right\rangle}$ with still the same averaging procedure and still excluding the $n=0$ modes. To have the magnetic correlation times, the fluctuating electrostatic potential is replaced by the magnetic one $\psi$ in the correlations function, and the Alfvens 
Table 4. Characteristic times of turbulence for electrostatic perturbations, with $\mathcal{K} u$ number for 3 cases: "interchange only" configuration at $\eta_{0} / 10$, "interchange + diamagnetic" configuration at $\eta_{0} / 10$ and the same configuration at $\eta_{0}$. The level of turbulent in $\%$ is also computed for each configuration..

\begin{tabular}{llll}
\hline & $\begin{array}{l}\text { Interch. } \\
\text { configuration at } \eta_{0} / 10\end{array}$ & $\begin{array}{l}\text { Interch.+ diamag. } \\
\text { configuration at } \eta_{0} / 10\end{array}$ & $\begin{array}{l}\text { Interch. + diamag. } \\
\text { configuration at } \eta_{0}\end{array}$ \\
\hline$\tau_{a c, E S}(s)$ & $5.27 \cdot 12^{-5}$ & $1.98 \cdot 12^{-4}$ & $2.32 \cdot 12^{-5}$ \\
\hline$\tau_{f, E S}(s)$ & $6.83 \cdot 12^{-5}$ & $2.67 \cdot 12^{-4}$ & $4.64 \cdot 12^{-5}$ \\
\hline $\mathcal{K} u(s)$ & 1.01 & 0.5 & 0.49 \\
\hline$\left.\frac{\delta p}{p}\right\rangle_{t}(\%)$ & 10.21 & 3.82 & 8.24 \\
\hline
\end{tabular}

speed is used for $v_{x}$.

The results are presented in table 4 . The characteristic times and resulting Kubo number $\mathcal{K} u$ for both configurations, interchange only and interchange + diamagnetic, are given for $\eta_{0} / 10$, resistivity at which the diamagnetic coupling matters (column 2 and column 3). Then, the impact of resistivity is shown in column 3 and 4 , where $\eta_{0} / 10$ is compared to $\eta_{0}$ for the interchange + diamagnetic configuration.

Both correlation times $\left(\tau_{a c, E S}\right.$ and $\left.\tau_{f, E S}\right)$ are increased when accounting for the diamagnetic coupling. Indeed the correlation length is higher when the diamagnetic effects are accounted for as the linear spectrum of this case was peaked at lower $k_{y}=k_{\theta} \rho_{i}$ than the spectrum of interchange only case [21]. The Kubo number is $\mathcal{K} u<1$ for all cases, meaning that the system is always in a hydro quasi-linear regime, explaining why figure 2 and figure 6 exhibit a similar trend. This result corroborates [17]. It also can be inferred from table 4 that a high Kubo number does not imply a high level of fluctuations, as $\langle\delta p / p\rangle_{t}$ decreases when the Kubo number increases. Values of $\langle\delta p / p\rangle_{t}$ corresponds to what can be found in tokamaks [29].

In this case, the equilibrium gradient $\left(g_{p}=1\right)$ is above the critical pressure gradient. This critical pressure gradient, above which instabilities appear, can be roughly defined by $g_{p, t h}=\left(\frac{\chi_{\perp} k_{\perp}^{3}}{\omega_{D} \kappa_{y}}\right)^{2}+\delta_{C}$, giving $g_{p, t h} \approx 0.1$ with parameters of table 1 . As $g_{p} \gg g_{p, t h}$, a diffusive transport is expected to dominate, as reported in [26]. In terms of Kubo numbers, a diffusive response corresponds to $\mathcal{K} u \ll 1$ while a ballistic response to $\mathcal{K} u \sim 1$ as explained in [26]. A scan in equilibrium gradient is performed to see if $\mathcal{K} u$ gets closer to 1 as we reduce the equilibrium gradient $g_{p}$ and results presented in figure 8 , reflecting the results of [26].

Gyro-kinetic non-linear simulations with GENE have not been performed here, but an on-going work using L-mode edge parameters can be [citation], and results are under publications. Very interestingly, those results also show the quasi-linear estimation of turbulent transport to be in agreement with that from non-linear simulations.

As explained in [27], a generic paradigm for reducing turbulent transport in the pedestal at the time of the transition in H-mode is based on sheared radial electric field. Indeed, a sheared radial electric field leads to a reduction of turbulent amplitudes, to a reduction of the phases between the fluctuations responsible for the turbulent 


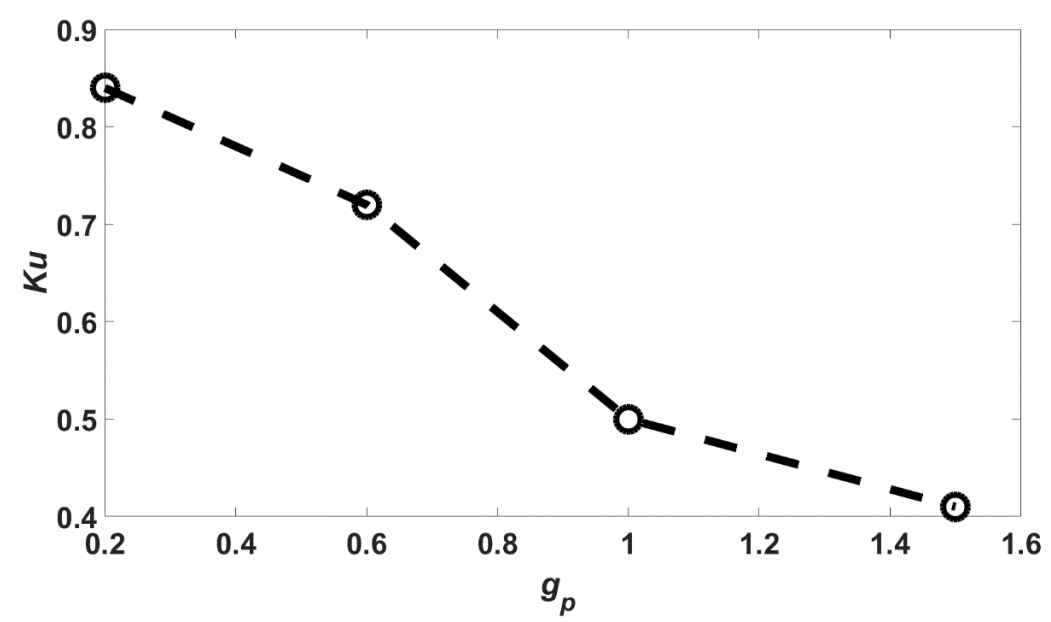

Figure 8. $\mathcal{K} u$ for different values of pressure gradient $g_{p} . \mathcal{K} u$ gets closer to 1 as the equilibrium gradient gets closer to the critical pressure gradient.

transport fluxes or to a reduction of correlation length and times. For the last case in particular, there is a criterion, known as the BDT (Bligari, Diamond, Terry) criterion, firstly derived to relate to the impact of the $\vec{E} \times \vec{B}$ shear on the radial correlation legnth $[28,39]$. However, it has been used in [38] showing that the impact of the $\vec{E} \times \vec{B}$ shear on the decorrelation time is much stronger. Then, one can observe turbulence suppression when:

$$
V_{\vec{E} \times \vec{B}}^{\prime}>\frac{1}{\Delta r k_{\theta} \tau_{c}}
$$

With $\tau_{c}$ the turbulence decorrelation time, $k_{\theta}$ the poloidal wave number, $\Delta r$ the correlation length. $V_{\vec{E} \times \vec{B}}^{\prime}=\frac{d V_{\vec{E} \times \vec{B}}}{d r}$ is the radial derivative of the $\vec{E} \times \vec{B}$ velocity. As $\frac{1}{\tau_{a c}} \approx \frac{1}{\Delta r k_{\theta} \tau_{c}}$ and $V_{\vec{E} \times \vec{B}}^{\prime} \approx \frac{1}{\tau_{\vec{E} \times \vec{B}}}$ turbulence suppression occurs when:

$$
\tau_{\vec{E} \times \vec{B}}<\tau_{a c}
$$

With $\tau_{a c}$ the auto-correlation time of the turbulence, which is the shortest between the particle flight time and the auto-correlation time. Therefore the parametric dependencies of both $\tau_{a c}$ and $\tau_{\vec{E} \times \vec{B}}$ matter in order to verify the BDT criterion and trigger a pedestal formation. The stabilizing impact of the diamagnetic coupling will ease this pedestal formation in our simulations. Also, as the resistivity is decreased, the L-mode edge turbulence drive is reduced, easing the ExB shear to quench the edge turbulence and lead to a pedestal formation. Therefore the impact of diamagnetic coupling on edge turbulence in our simulations can be summarized by these points:

- It increases the linear growth rate but reduces the phase shift and then the proxy of the flux, especially at low resistivity 
- This trend is confirmed nonlinearly

- $\tau_{a c}<\tau_{f}$ and the nonlinear phase shift stays close to the linear one, then the quasilinear approximations is valid.

\section{Impact of magnetic fluctuations in L-mode edge plasma}

The net transport due to magnetic fluctuations in tokamak edge turbulence is found to be very weak if we compare it to the convective transport due to $\tau_{\vec{E} \times \vec{B}}$ motion at low $\beta$ values characteristic of tokamak L-mode edge (see tables 1 and 2) [14]. Nonetheless, magnetic fluctuations can have two distinct effects: they are responsible for the magnetic transport, mentioned earlier, and they modify the stability of unstable modes and subsequently the convective $\tau_{\vec{E} \times \vec{B}}$ transport. In this section, the second point will be studied. The impact of $\beta$ will be first studied linearly, with EMEDGE3D and GENE. A criteria for the impact of $\beta$ will be first derived, showing that it is more destabilizing for low values of $k_{y}=k_{\theta} \rho$ and resistivity, which is then confirmed by EMEDGE3D and GENE linearly. This is also confirmed using nonlinear simulations with EMEDGE3D. Nonetheless, the quasilinear character of the underlying dynamic is still valid for any values of $\beta$ and correlation times are decreased when $\beta$ is increased. Finally, the contributions of the Reynolds stress and the Maxwell stress to the $\vec{E} \times \vec{B}$ will be shown to increase with $\beta$, the contribution of the Maxwell stress remaining marginal.

\subsection{Impact on linear instabilities}

Numerous MHD models are using an electrostatic assumption as in [30,31]. Indeed, taking the values for a typical JET-like $\mathrm{L}$ mode pulse seen in table 2 , we find $\beta \approx 0.16 \%$ which is quite low. In fact the critical $\beta$ for ideal MHD is proportional to the gradient length $\beta_{c} \sim L_{p} / R_{0}$. Indeed, the equilibrium with the divergence of the diamagnetic current is:

$$
\tilde{j}_{\|} \sim k_{\theta} \tilde{p}
$$

As a first approximation, the pressure comes from the relation $\nabla_{\|} p=0$ along the perturbed field lines, hence:

$$
\tilde{p} \sim \frac{\frac{q R_{0}}{B_{0}} k_{\theta} \bar{p} \tilde{A}_{\|}}{L_{p}}
$$

Therefore:

$$
\mu_{0} \tilde{j}_{\|} \sim \frac{k_{\theta}^{2} \mu_{0}}{B_{0}^{2}} R_{0} \frac{d \bar{p}}{d r} \tilde{A}_{\|}
$$


The Amperes law leads to $k_{\perp}^{2} \tilde{A}_{\|} \sim \mu_{0} \tilde{j}_{\|}$or in other words $\frac{\beta_{c} R_{0}}{L_{p}} \sim 1$. In the case $\beta>\beta_{c}$ the Kinetic Ballooning modes are destabilized [33]. This value of $\beta_{c}$ is usually high in core plasma. But taking the parameters of table 1 corresponding to edge plasmas, $\beta_{c} \sim 1.6 \%$. Then even if $\beta$ for L-mode parameters is low, the fact that $R_{0} / L_{p}$ is very large (64 here but can be up to 120 [5]) leads to low $\beta_{c}$. Hence, magnetic effects matter at the edge; these effects are studied in details in this section.

To know the parametric dependencies of the impact of $\beta$, one can study the competition between the resistive parallel current $\eta \tilde{j}_{\|}$and the induced parallel current $\partial_{t} \tilde{A}_{\|}$. This can be seen by comparing the terms in the Ohms law:

$$
\partial_{t} \tilde{A}_{\|}=-\nabla_{\|}\left(\tilde{\phi}-\tilde{p}_{e}\right)+\eta \tilde{j}_{\|}
$$

This gives:

$$
\tilde{j}_{\|}=-\frac{i\left[\omega \tilde{A}_{\|}-k_{\|}\left(\tilde{\phi}-\tilde{p}_{e}\right)\right]}{\eta}
$$

Using the Amperes law: $\nabla_{\perp}^{2} \tilde{A}_{\|}=-\mu_{0} \tilde{j}_{\|}$and decomposing the oscillation frequency $\omega$ in its real part $\omega_{R}$ and its imaginary part $\gamma\left(\omega=\omega_{R}+i \gamma\right)$ implies that the impact of $\beta$ is weak if the induced current is much lower than the resistive current:

$$
\gamma \tilde{A}_{\|} \ll \eta \tilde{j}_{\|}
$$

Hence if:

$$
\gamma \ll \frac{\eta}{\mu_{0}} k_{\perp}^{2}
$$

At a given resistivity, the effect of larger $\beta$ on instabilities depends on $k_{\perp}$. Figure 9 represents the ratio between the maximum growths rates for 2 values of $\beta$ : $\beta=0.32 \%$ (as given in table 1 ) and ten times lower: $\beta / 12=0.032 \%$, for each toroidal mode number $n$. For our modal resolution $\left(n_{\max }, m_{\max }\right)=(32,132)$ (then $\left.k_{\perp}<0.26\right) \beta$ is always destabilizing, especially for the lowest wavenumber as represented in equation 8 , i.e where the induced parallel current is decreased.

In addition to $\beta$, the limit also depends on the resistivity. At a given wave number $k_{y}=k_{\theta} \rho$, the impact of $\beta$ is stronger for low values of resistivity. Recalling from section 2 that the resistivity is critical in determining the dominant instability and is stabilizing. There are then two opposite effects of the resistivity, and surely interplay between them.

The impact of $\beta$ is now linearly studied with GENE and using table 2 parameters. The normalized resistivity is $\frac{\nu}{C_{s} / R}=1.3$ for both cases and figure 12 (a) shows the growth rates versus $\beta$ at $k_{\theta} \rho=0.1$ while figure $12(\mathrm{~b})$ at $k_{\theta} \rho=0.5$. It can be observed that $\beta$ has a destabilizing effect, which is stronger at $k_{\theta} \rho=0.1$ than at $k_{\theta} \rho=0.5$. 


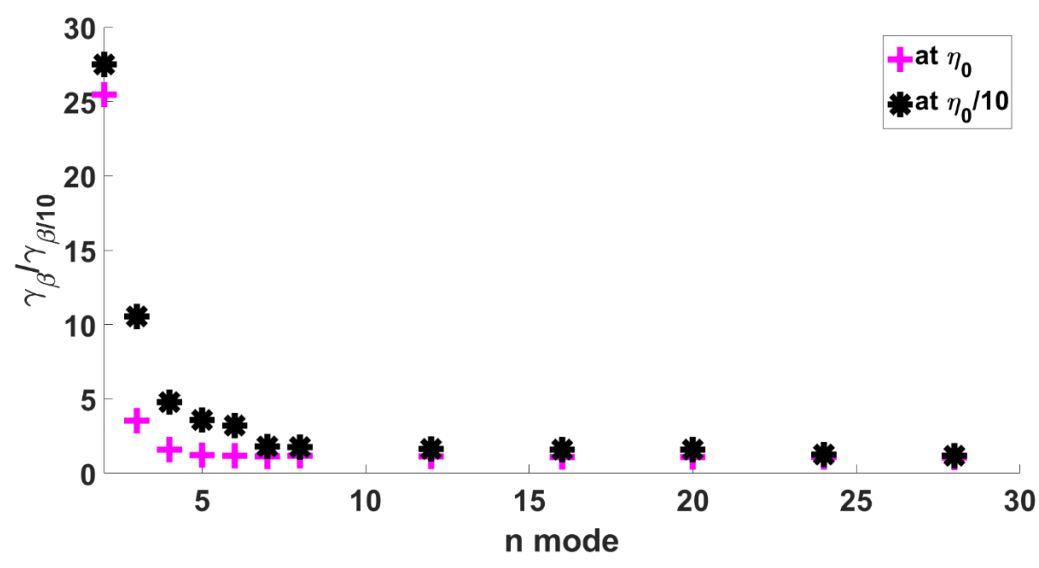

Figure 9. Ratio of maximum growth rate $\gamma_{\beta} / \gamma_{\beta / 12}$ for each toroidal mode, for two values of resistivity. The effect of $\beta$ is higher at low resistivity and for low $k_{\theta} \rho$.

Indeed, for the lowest value of $k_{\theta} \rho$, the growth rate increases up to a factor 2 between $\beta_{e}=1.6 \cdot 12^{-3}$ where $\gamma \tau=2.3$ and $\beta_{e}=4.612^{-3}$ where $\gamma \tau=4.9$. At $k_{\theta} \rho=0.5$, between the same values of $\beta_{e}, \gamma \tau$ increases only from 3.1 to 3.6 , then it is increased by a factor 0.86 .
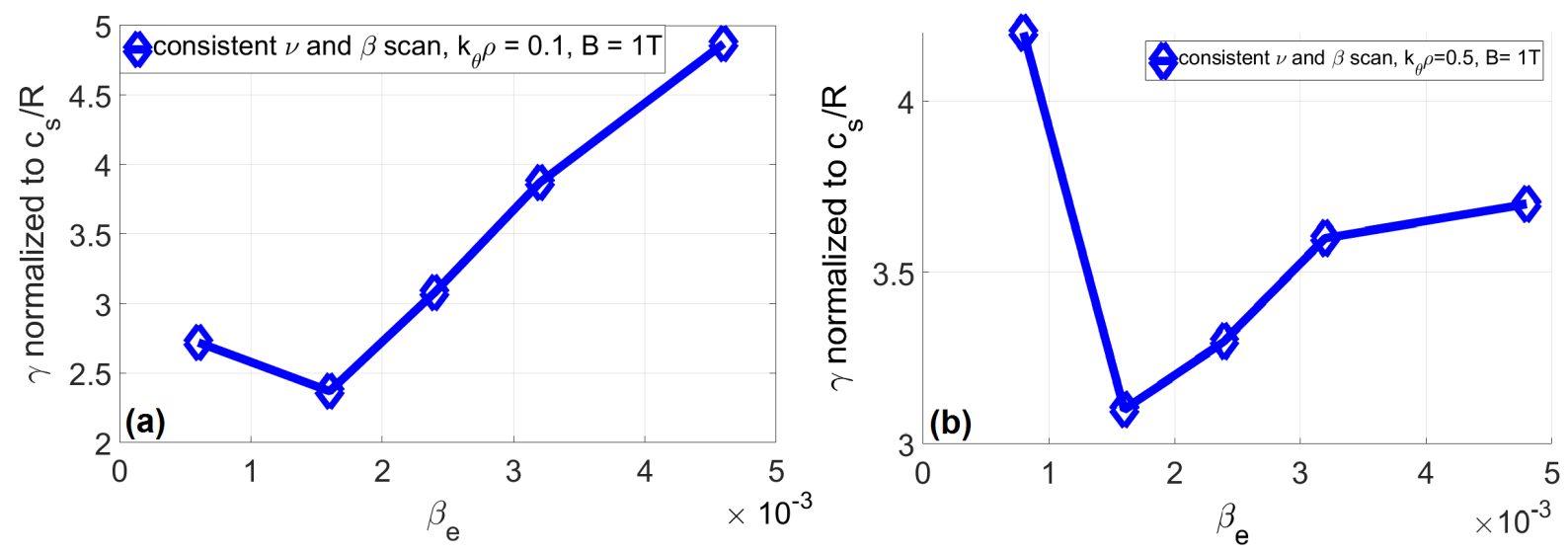

Figure 10. (a) Linear growth rate with GENE for $k_{\theta} \rho=0.1$, as a function of $\beta_{e}=\beta / 2$ at $\frac{\nu}{C_{s} / R}=1.3\left(T_{e}=50 \mathrm{eV}\right)$ (b) Linear growth rate with GENE for $k_{\theta} \rho=0.5$, as a function of $\beta_{e}=\beta / 2$ at $\frac{\nu}{C_{s} / R}=1.3$.

\subsection{Impact of $\beta$ in nonlinear fluid simulations using EMEDGE3D}

In nonlinear fixed gradient EMEDGE3D simulations, the destabilizing effect of larger $\beta$ is recovered for the modal resolution chosen $\left(k_{y}=k_{\theta} \rho \in[0 ; 0.26]\right)$. Here we also account for the diamagnetic coupling as we have shown it was critical at realistic resistivity. The simultaneous stabilizing impact of diamagnetic coupling, with the destabilizing impact of increased $\beta$ has never been isolated before. Indeed in [12] the diamagnetic coupling is taken into account and discussed with an electromagnetic model, but the 
effect of $\beta$ was not isolated. Figure 11 (a) represents the nonlinear turbulent flux $Q_{E}$ (see section 2) as a function of resistivity for three values of $\beta$. The solid black line represents the $2 \beta=0.64 \%$ case. For this larger value of $\beta$, the resistivity is only very slightly destabilizing. The blue dashed line represents $\beta=0.32 \%$, where the nonlinear turbulent flux increases by an order of magnitude when increasing from $\eta_{0} / 10$ to $\eta_{0}$. Finally, the red dotted line represents $\beta / 10=0.032 \%$, where the nonlinear turbulent flux increases by 4 decades when ramping up the resistivity from $\eta_{0} / 10$ to $\eta_{0}$. The destabilizing impact of $\beta$ is stronger at the lowest resistivity where the diamagnetic coupling is the most stabilizing. On figure 11 (b) phase shifts spectrum are presented for each value of $\beta$ at the nominal resistivity $\eta_{0}$. The phase shift is increasing when $\beta$ is increased.
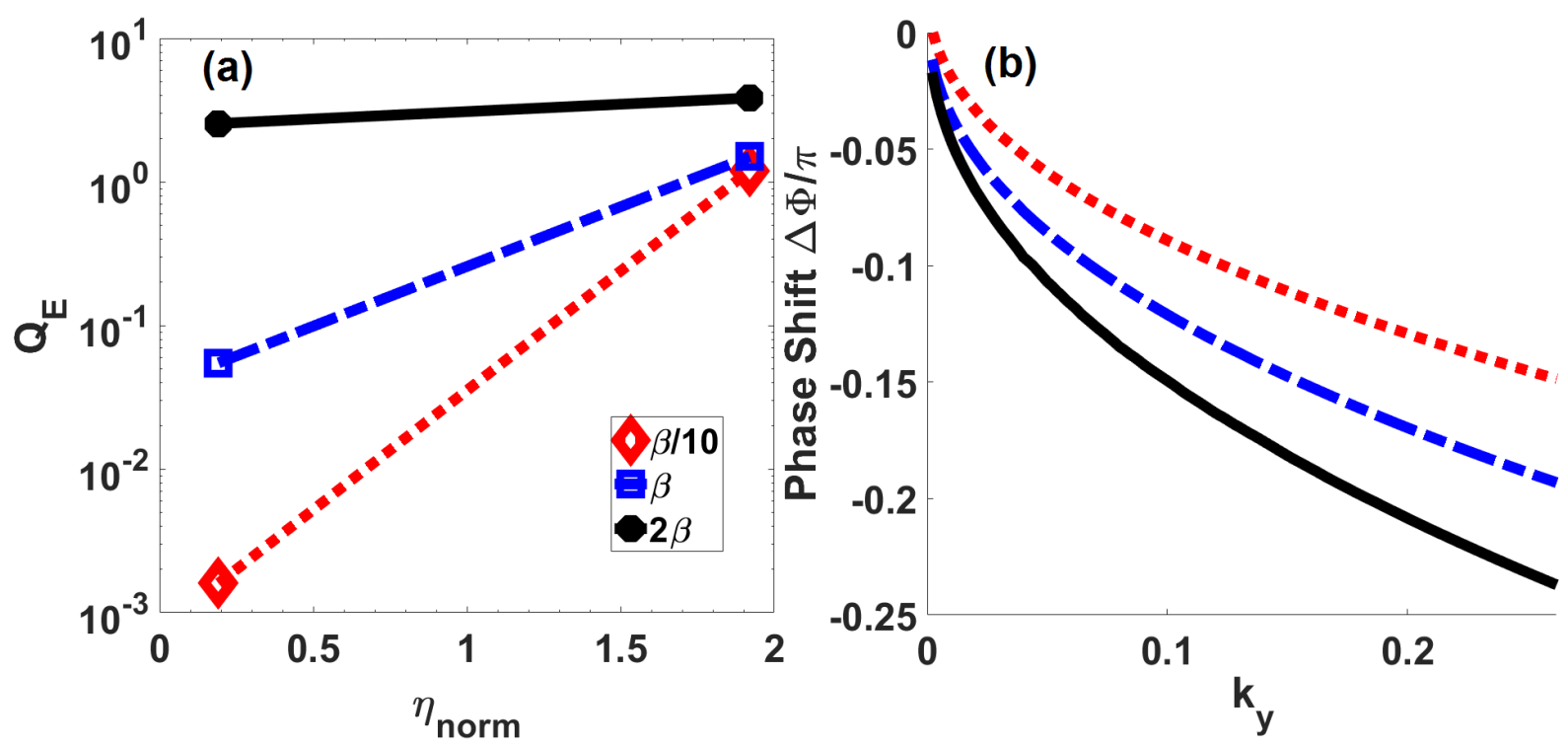

Figure 11. (a) Nonlineear turbulent flux $Q_{E}=\left\langle p_{n m} \partial_{y} \phi_{n m}\right\rangle$ with respect to resistivity for $\beta / 12, \beta, 2 \beta$ computed with EMEDGE3D in a non-linear case using parameters of table 1. (b) Phase shifts spectrum for the different values of $\beta$ at the nominal resistivity $\eta_{0}$.

With this modal resolution limited to $k_{y}=k_{\theta} \rho<0.26, \beta$ is always destabilizing. Note that, in experiments, when the power is increased, $\beta$ increases and $\eta$ decreases, the first being destabilizing and the latter being stabilizing, likely resulting in a competition between the two effects. Increasing $\beta$ also has an effect on reducing both the electromagnetic auto-correlation time $\tau_{a c, E M}$ and the particle flight time $\tau_{f, E M}$, defined in section 2.3 as summarized in table 5 .

In Table 5 are given $\tau_{a c, E S}$ and $\tau_{f, E S}$ for electrostatic fluctuations and $\tau_{a c, E M}$ and $\tau_{f, E M}$ for magnetic fluctuations given by EMEDGE3D nonlinear fixed gradient simulations using the parameters of table 1 and three values of $\beta$. All correlation times decrease with $\beta$, which is expected. The flight time for magnetic perturbations also decreases with $\beta$ as the Alfven speed increases. Speaking in terms of Kubo number defined in section 2.3 , one gets $1 \sim \mathcal{K} u_{2 \beta}>\mathcal{K} u_{\beta}>\mathcal{K} u_{\beta / 10}$. Therefore the quasi-linear 
Table 5. $\tau_{a c}, \tau_{f}$ and $\mathcal{K} u$ for electrostatic and magnetic perturbations, at three different $\beta$. The times used to compute the Kubo number are highlighted. The inverse of the linear growth rate is also shown to decrease with increasing $\beta$.

\begin{tabular}{llll}
\hline & $2 \beta$ & $\beta$ & $\beta / 12$ \\
\hline$\tau_{a c, E S}(s)$ & $2.27 \cdot 12^{-5}$ & $2.32 \cdot 12^{-5}$ & $2.67 \cdot 12^{-5}$ \\
\hline$\tau_{a c, E M}(s)$ & $2.05 \cdot 12^{-5}$ & $3.01 \cdot 12^{-5}$ & $3.29 \cdot 12^{-5}$ \\
\hline$\tau_{f, E S}(s)$ & $3.47 \cdot 12^{-5}$ & $4.64 \cdot 12^{-5}$ & $5.42 \cdot 12^{-5}$ \\
\hline$\tau_{f, E M}(s)$ & $2 \cdot 12^{-5}$ & $4.83 \cdot 12^{-5}$ & $5.54 \cdot 12^{-4}$ \\
\hline $1 / \gamma_{\text {lin }}(s)$ & $0.92 \cdot 12^{-5}$ & $1.21 \cdot 12^{-5}$ & $1.31 \cdot 12^{-5}$ \\
\hline $\mathcal{K}$ & 1.01 & 0.5 & 0.49 \\
\hline
\end{tabular}

regime is valid for all $\beta$ and even more so as $\beta$ gets lower [18]. But it is really close to 1 for the $2 \beta=0.64 \%$ case. This means that for the highest values of $\beta$, the quasilinear approximation is less valid. It also can be seen on the inverse of the growth rate, which is the farther from the correlations times in the $2 \beta=0.64 \%$ case.

We recall that the BDT criterion, defined in section 2.3 [28] is applied on the decorellation time as suggested in [38] rather than on the radial correlation length [39]. Here, as the correlation times are reduced as $\beta$ increases, it could more difficult to stabilize the L-mode edge turbulence by $\vec{E} \times \vec{B}$ shearing as $\beta$ gets higher. This cannot be seen only looking at the transport equations.

On the other hand, the contribution of the Reynolds and Maxwell stresses to the $\vec{E} \times \vec{B}$ shear flow can be deduced from the transport equation 2. Their derivation can be found in [14], and recalled here in equation 9:

$$
\partial_{t}\left\langle u_{\vec{E} \times \vec{B}}\right\rangle=-\left\langle\tilde{u}_{r} \tilde{u}_{\theta}\right\rangle+C_{1} \beta_{e}\left\langle\tilde{b}_{r} \tilde{b}_{\theta}\right\rangle+\omega_{D}\langle p \sin \theta\rangle+\partial_{r}\left\langle\nu_{\perp} \partial_{r} u_{\vec{E} \times \vec{B}}\right\rangle
$$

With $\tilde{u}_{r}=\partial_{\theta} \tilde{\phi}, \tilde{u}_{\theta}=-\partial_{r} \tilde{\phi}, \tilde{b}_{r}=\partial_{\theta} \tilde{\psi}, \tilde{b}_{\theta}=-\partial_{r} \tilde{\psi}, \omega_{D}\langle p \sin \theta\rangle$ the contribution of the curvature (not analyzed here) and $\partial_{r}\left\langle\nu_{\perp} \partial_{r} u_{\vec{E} \times \vec{B}}\right\rangle$ and damping term. Hence, we look at the impact of $\beta$ on the Reynolds and the Maxwell stresses. The Reynolds stress is defined as $R S=\left\langle\left\{\phi, \nabla_{\perp}^{2} \phi\right\}\right\rangle=\left\langle\tilde{u}_{r} \tilde{u}_{\theta}\right\rangle$ and the Maxwell stress is defined as $M S=\left\langle\left\{\psi, \nabla_{\perp}^{2} \psi_{\perp}\right\}\right\rangle=C_{1} \beta_{e}\left\langle\tilde{b}_{r} \tilde{b}_{\theta}\right\rangle$. In electrostatic models, the Maxwell stress is neglected. Then, the Reynolds stress can act freely to help the neoclassical friction to form a pedestal $[15,16]$. But in electromagnetic simulations, the Maxwell stress can reduce and even cancel the effects of the Reynolds stress.

Therefore, both stresses are computed and represented in figure 12 . Figure 12 (a) represents the Maxwell stress with respect to $\beta$, in arbitrary units. Figure 12 (b) represents the Reynolds stress in arbitrary units as a function of $\beta$. They are both plotted along with their signs and coefficients, a negative sing implying that the stress participate to the $\vec{E} \times \vec{B}$ flow. It can be seen that both stresses increase (in absolute value) as $\beta$ increases. This means that the Reynolds stress is a greater source for the $\vec{E} \times \vec{B}$ flow as $\beta$ grows, whereas the Maxwell stress is a greater sink for the flow as $\beta$ increases. Finally the fluctuations of the stresses increases with $\beta$. If the Maxwell stress 
fluctuates more than the Reynolds stress at high $\beta$, its contribution to the flows remains small compared to the Reynolds stress.

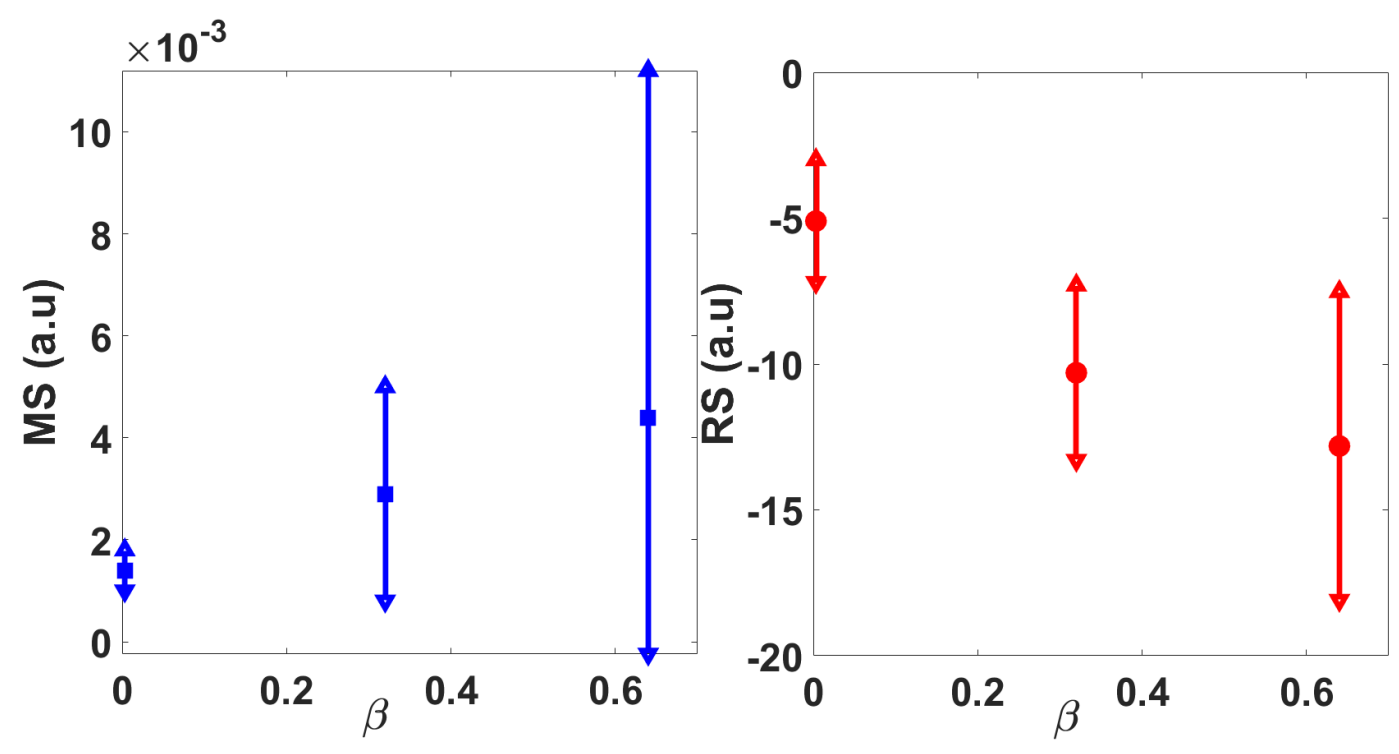

Figure 12. (a) Reynolds stress (a.u) with respect to time normalized to $L_{p} / c_{s}$ (b) Maxwell stress with respect to time. From left to right, for (a) and (b): $2 \beta, \beta$ and $\beta / 12$ cases.

Nevertheless, as expected by a reduction of the stabilizing impact of the induced current, $\beta$ has a destabilizing effect in our fluid simulations, especially when it grows from low to moderate values. The growth rate and the nonlinear turbulent flux increase as the resistivity is decreased and especially at low $k_{\theta} \rho$ for the growth rate. The phase shift is also increased when $\beta$ is increased. If the quasilinear approximation is still valid here at any values of $\beta$, increasing $\beta$ leads to lower correlation times. It should be then more difficult for the $\vec{E} \times \vec{B}$ shear to tilt the turbulent eddies, and then to trigger the $\mathrm{L}-\mathrm{H}$ transition at high $\beta$. On the other hand, the contribution of the Maxwell stress is negligible compared to the contribution of the Reynolds stress. Even if the Maxwell stress is a sink for the flow, it should not impact it a lot.

\section{Conclusion}

In this work, the nature of the turbulent transport for realistic L-mode edge parameters prior to a transition in $\mathrm{H}$-mode has been investigated. The gyrokinetic code GENE has been used linearly and the fluid code EMEDGE3D had been used linearly and nonlinearly in this purpose. As previously demonstrated, the diamagnetic coupling has been shown to be stabilizing especially at low and more realistic resistivity in our simulations, and on the other hand, larger $\beta$ is destabilizing.

It has been demonstrated with the gyro-kinetic code GENE and the fluid code EMEDGE3D that linear unstable modes depend on the resistivity in our simulations. At low edge like values of resistivity, there is a competition between the resistive ballooning 
modes and resistive drift waves. If the growth rate increases when the diamagnetic coupling is accounted, the phase shift between the pressure and the electrostatic potential decreases, leading to a less efficient transport. The nonlinear turbulent flux computed with EMEDGE3D is also lower when diamagnetic coupling is accounted for, especially at low and more realistic resistivity. Increased $\beta$ is shown to be destabilizing at realistic resistivity especially at low $k_{y}=k_{\theta} \rho$ in these simulations: both the growth rate and the turbulent transport are increased when $\beta$ is increased. This means that special care is required when hypothesis for the model are made for edge-like parameters. The contribution of the Reynolds and Maxwell stresses on the $\vec{E} \times \vec{B}$ flows increase when $\beta$ is increased. The Reynolds stress acts as a source while the Maxwell stress acts as a sink. On the other hand, the contribution of the Maxwell stress is negligible compared to the contribution of the Reynolds stress. Even if the Maxwell stress is a sink for the flow, it should not impact it a lot. Nevertheless, the impact of $\beta$ is more important on the characteristic times of the turbulence than on transported quantities.

Moreover it is shown with the nonlinear simulations that theauto-correlation time is lower than the particle flight time. The linear and nonlinear phase shifts are also very similar, demonstrating that L-mode edge turbulent transport is quasilinear at various values of resistivity and $\beta$ in EMEDGE3D simulations. This allows the development of a reduced quasi-linear model adapted to the L-mode edge region. Since $\beta$ leads to lower correlation times, it should be then more difficult to trigger the L-H transition at high $\beta$. This corroborates the dependence of a normalized power threshold with $\beta$, which is increasing with $\beta$.

Taking this study into account, further flux driven simulations can be performed with EMEDGE3D to investigate the power threshold required to find an auto-consistent formation of a transport barrier at the very edge of the plasma, as previously observed in [13] with the electrostatic version of the model [13].

\section{Acknowledgments}

The authors acknowledge fruitful discussions with the DSC team at the PIIM UMR 7345 in particular with O. Aguillo, M. Muraglia and Y. Camenen, and also to the members of the GMICS group, especially to J. Morales. An additional special thanks to J. Citrin for his help to the calculation of correlation functions. The authors would thank all the JET team for sharing L-H transition parameters. A special thanks to Nicola Bonanomi for sharing its work with GENE, as an element of comparison. This work has been carried out within the framework of the EUROfusion Consortium and has received funding from the European research and training program 2014-2018 and 2019-2020 under grant agreement n 633053. The views and opinions expressed herein do not necessarily reflect those of the European Commission.

\section{References}

[1] Ryter F. et al, H mode power threshold database for ITER, Nucl. Fusion 361217 (1996). 
[2] Takizuka T. and ITPA H-Mode Database Working Grp, Roles of aspect ratio, absolute B and effective $\mathrm{Z}$ of the H-mode power threshold in tokamaks of the ITPA database, Plasma Phys. Control. Fusion 46 A22733 (2004)

[3] Martin Y.R., Takizuka T. and the ITPA CDBM H-mode Threshold Database Working Group, J. Phys.: Conf. Ser. 123012033 (2008)

[4] C. Bourdelle, L. Chn, N. Fedorczak, X. Garbet, , Nucl. Fusion 55073015 (2015)

[5] C. Bourdelle, X. Garbet, R. Singh and L. Schmitz, Plasma Phys. Control. Fusion 54115003 (2012)

[6] C. Bourdelle, Nucl. Fusion 54022001 (2014)

[7] A. Hasegawa and K. Mima, Phys. Rev. Lett. 39205 (1977)

[8] A. Zeiler and D. Biskamp, Physics of Plasmas 52654 (1998)

[9] B. Scott, Plasma Phys. Control. Fusion 39471 (1997)

[10] B.Scott, Physics of Plasmas 71845 (2000)

[11] B. Scott, Physics of Plasmas 12062314 (2005)

[12] A.H. Nielsen, J. Madsen, G. Xu, V. Naulin, J. Juul Rasmussen, N. Yan, 2d fluid simulations of interchange turbulence with ion dynamics, 40th European Physical Society Conference on Plasma Physics, Espoo, Finland (2013)

[13] P.Tamain et al, Journal of Computational Physics 229361378 (2012)

[14] G. Fuhr, P. Beyer, S. Benkadda, and X. Garbet, Phys. Rev. Lett 12119500 (2006)

[15] L. Chôné et al, Physics of Plasmas 21070702 (2014)

[16] L. Chôné et al, Nucl. Fusion 55073012 (2015)

[17] J.Citrin, Physics of Plasmas 19062305 (2012)

[18] J. Krommes, Phys. Rep. 3601 (2002)

[19] T. Dannert and F.Jenko, Physics of Plasmas 12072309 (2005)

[20] C.Bourdelle, Plasma Phys. Control. Fusion 58014036 (2016)

[21] Y. Sarazin, Habilitation diriger des recherches,(2008)

[22] F. Jenko, Comput. Phys. Commun. 125196209 (2000)

[23] S. Brunner, T. Dannert, F. Jenko, F. Merz, T. Grler, X. Lapillonne and D. Told, J. Comput. Phys $2307053(2011)$

[24] R. Kubo, J. Math. Phys. 4174 (1963)

[25] Z. Lin, Phys. Rev. Lett. 99265003 (2007)

[26] X. Garbet, Y. Sarazin, F. Imbeaux, P. Ghendrih, and C. Bourdelle, Physics of Plasmas 14122305 (2007)

[27] J. W. Connor and H. R. Wilson, Plasma Phys. Control. Fusion 42 R1 (2000)

[28] Biglari, Diamond and Terry, Phys. Fluids B 21 (1990)

[29] R. Sabot, A. Sirinelli, J.-M. Chareau J.-C. Giaccalone, a dual source D-band reflectometer for density profile and fluctuations measurements in Tore-Supra, Nucl. Fusion 465685 (2006)

[30] P.Tamain et al, Journal of Computational Physics 229 361-378 (2012)

[31] G. Y. Park, S. S. Kim, Hogun Jhang, P. H. Diamond, T. Rhee, and X. Q. Xu, Physics of Plasmas 22032505 (2015)

[32] A. Ishizawa, N. Nakajima, Nucl. Fusion 49055015 (2009)

[33] G. Rewoldt, W.M. Tang, R.J. Hastie, Phys. Fluids 30807 (1987)

[34] X. Garbet, Habilitation diriger des recherches (2009)

[35] P. Manz, Physics of Plasmas 19072311 (2012)

[36] K. Miki, Physics of Plasmas 19092306 (2012)

[37] F. Jenko et al, Plasma Phys. Control. Fusion 47 B195 (2005)

[38] G. M. Staebler et al, Phys. Rev. Lett. 110055003 (2013)

[39] T. S. Hahm, Phys. of Plamas 21648 (1995) 